\title{
Building Capacity in Program Practitioner Realist Evaluation Through Application of CAE Principles
}

Kevin Harris Ph.D.

Department of Sport Health and Social Sciences, Southampton University, UK

\author{
For Ethan
}

June 2018

To appear in J. B. Cousins (Ed.). Global Test Drive of Principles for Collaborative Approaches to Evaluation (CAE) Thousand Oaks: Sage 


\begin{abstract}
Sport for Development (SfD) projects are under close scrutiny, concerning the capacity that sport has to create social change. Critics have argued that understanding 'what works for whom and why' is required when evaluating SfD projects. This chapter explores stakeholder involvement in evaluation, mobilising an Integrated Instructional Framework (IIF) with emerging SfD practitioners which incorporates elements of both Shulha et al's (2016) principles to guide collaborative approaches to evaluation (CAE) and realist evaluation (Pawson \& Tilley, 1997). Specifically, 15 practitioners participated encompassing 5 community focused SfD projects delivered within an innovation program at a university in the United Kingdom. The IIF was evaluated incorporating Q-method factor analysis. Findings on the value of the IIF for practitioners emerged. Four groups of practitioners emerged depicting how the CAE principles applied to enable the practitioners to mobilise realist evaluation. These groups were 'new and emerging evaluators', 'polished problem solvers', 'passive passengers', and 'proficient yet sceptical practitioners'. These were described using holistic narratives demonstrating shared viewpoints about the IIF and the relevance of the CAE principles. In conclusion, the CAE principles embedded within the IIF were pivotal to enable emergent practitioner evaluation capacity development.
\end{abstract}




\section{Introduction}

Sport for Development (SfD) projects (also referred to as interventions, and initiatives) have proliferated across the globe in the last twenty years. SfD projects have used sport as a tool to address a wide range of different personal and social outcomes, such as the use of sport / physical activity to empower marginalized populations (Mwaanga 2011), promote self-esteem, tackle health and inactivity (Weed 2016), build community cohesion (Adams 2008) and tackle anti-social behaviour (Crabbe 2007). Moreover, SfD has taken on a considerable role in social issues agendas. At present, and particularly within the UK, there has been a shift to combine sport and public health to address issues of physical inactivity and obesity (Weed 2016).

A lack of evidence discourse currently exists in respect of the capacity of sport to address wider social goals (Nichols et al. 2011). Coalter (2007, 2010) has emphasised the limited evidence base of SfD, highlighting the prevalence of ill-defined programmes with hard to follow outcomes as well as poor evaluation practice. Thus, a field which is defined by its claims rather than its results has emerged and sceptics have questioned the given assumption that sport can solve a multitude of problems (Coalter 2007, 2010; Kay 2009; Levermore 2011). In response to the lack of evidence, a significant increase in the use of evaluation approaches has taken place (see Harris, 2018); yet the question 'Does sport work?' is firmly rooted in SfD's approach to evidence. Funders and politicians who invest money and resource into SfD want to see results and impact, thus it is of little surprise that the point of departure for any approach to evaluation may be to find evidence to support positive outcomes, or show proof) that SfD works (Harris \& Adams, 2016; Smith \& Leach, 2010).

Key issues remain in understanding how, why, and for whom projects and programs create outcomes (Pawson \& Tilley 1997). Harris and Adams (2016) have called for approaches that focus on Pawson and Tilley's (1997) 'realist evaluation' to understand how and why interventions work. Realist evaluation has developed specific momentum across a wide variety of sectors in the last 10 years; specifically within the healthcare and nursing sector (Abhyanker et al. 2013), but also in participatory settings (Jagosh et al. 2012). However, its mobilisation within SfD has been limited at best. Aside from Nichols (2007) and Duffy et al. (2013), very few evaluators have attempted to apply realist evaluation and whilst participatory approaches to evaluation that may mobilise theory of change exist (Levermore 2011), there have been few attempts to train SfD practitioners to carry out realist or theory driven approaches within their work. Reasons for this may be connected to the 
traditional SfD evaluation approaches which require that outcomes are captured as opposed to the processes that lead to them (Coalter 2007).

SfD practitioners are rarely involved in mobilising evaluation work (Harris \& Adams 2016). Many of these approaches are carried out either independently by academics (Kay 2012) or within participatory frameworks that may involve program practitioners to different extents (Levermore 2011). Involving practitioners more collaboratively in this work has potential to develop the evidence base of SfD because practitioners are situated within their project contexts and are well placed to interpret and reflect upon the extent to which any change is manifesting itself. Deeper practitioner engagement with evidence-based practice may help to address many of the subjugating power imbalances omnipresent in multiple evaluation approaches (Harris \& Adams 2016; Nichols et al. 2011) because it places value upon their role and their insight. Whilst the enactment of evaluation requires a certain competency which practitioners may not possess due to limited training (Hylton \& Hartley 2011), this can be mitigated providing that appropriate capacity building and training is implemented (Fetterman 2001).

In this chapter, I draw upon findings that emerged following my implementation of an Integrated Instructional Framework (IIF) which incorporates elements of both Shulha, Whitmore, Cousins, Gilbert and Al Hudib's. (2016) principles to guide collaborative approaches to evaluation (CAE) and realist evaluation (Pawson \& Tilley, 1997). In my role as course leader and tutor I developed the IIF to support emerging undergraduate student SfD practitioners $(\mathrm{N}=15)$ in a higher education context at a university in the south of the UK. I supported them to use realist evaluation to evaluate their own SfD projects that they were developing, delivering and evaluating in local communities. Within a context of limited understanding of evaluation practice amongst the SfD practitioners, I saw value in applying the CAE principles with realist evaluation as it could help determine how and why projects were successful (Pawson \& Tilley 1997). My shaping of the IIF for increasing realist capacity was premised upon the importance for establishing motivation for collaboration and in order to understand to what extent there was intent to learn realist evaluation. I wanted to consider how meaningful relationships between myself and the 15 practitioners could enable an appropriate participatory and collaborative process to be mobilised, and to understand to what extent this evaluation practice could promote evaluative thinking and enhance use of the findings to support the sustainability of the practitioners' future SfD projects. These aspirations are congruous with the goals of the CAE principles and this was a key reason for their application in the IIF. In essence, my key aim was to leverage evaluation capacity 
building among SfD practitioners in ways other conventional approaches (Levermore 2011) have not. My test drive of the CAE principles would enable me to assess how well (or not) they worked for this purpose.

This chapter is made up of the following structure. I will explain the context for my field test of the IIF, which in short is a higher education environment in the UK where emerging SfD practitioners develop, deliver and evaluate their own SfD projects. I will then describe my development of the IIF and how I applied it in practice drawing upon its key components and how realist evaluation and CAE principles were integrated. Having provided a context and description of the IIF I then explain how I evaluated it through the use of Q methodology (Watts \& Stenner 2012) which uncovered four different groups within the 15 student SfD practitioners engaging within the IIF. I will conclude with an overall discussion concerning the contribution of the CAE principles to the IIF.

\section{Context}

The IIF was piloted within the Coaching Innovation Program (CIP) at a university in the south of the UK. The CIP is student-led, curriculum-based program enabling small groups of student practitioners (usually comprising of 3-4 students) to research the needs of a community and develop their own SfD project with local stakeholders. These projects fit the context of SfD, and currently reflect a significant volume of activity in the area, reaching one thousand beneficiaries each year and spanning sport and public health agendas. The projects are developed over an entire academic year (eight months). Initially, students (hereafter 'practitioners') work to build a realist program theory - or a set of assumptions and beliefs around how and why their projects will work - through establishing need with stakeholders. The practitioners deliver their project and evaluate it in the following academic year. However, at the time of mobilising the IIF, the CIP did not have a substantial approach to evidence which could have left it vulnerable to the same critiques already cast upon the SfD field.

I offered the opportunity to voluntarily opt into the IIF which was an added extra to the curriculum to five diverse projects each of which accepted:

- Edumaths: A physical activity project delivered in a local primary school to address physical literacy and bring movement-based education into the maths curriculum. 
- Heart of the Community: A chair based physical activity project designed to tackle isolation and loneliness for over 60's.

- Gateway: A college based project working with less academic young people using sport to develop employability skills.

- Mums on the Move: A physical activity project using Zumba to encourage sedentary mums living in a deprived area to get active.

- Mentor Me: A project in a local secondary school designed to train pupils aged 15-16 years to coach and mentor younger peers disengaged from Physical Education.

All of the practitioners in these projects had little or no experience in evaluation work or data collection and research. Their provisional motivation for collaboration was premised upon the intention to learn more about evaluation practice to enhance the understanding they could glean from evaluating their project. They were also motivated to do well in the curriculum. I was directly involved within the IIF. I created and managed the curriculum mobilising the CIP alongside a number of other academics.

\section{Development of the IIF}

I created the IIF to place the practitioner at the centre of mobilising evaluation which is rare within SfD (Harris, 2018). I also wanted to address the limited insight we have in social programs for understanding how and why programs may (or not) work (Pawson \& Tilley, 1997). Realist evaluation emerged as an appropriate approach to address this gap (Harris 2018), yet I recognised how important it would be to embed a collaborative evaluation culture where support, ownership and guidance could be fostered, so that the practitioners could grasp (based on their limited experience) key terms. I will now justify realist evaluation's application in $\mathrm{SfD}$, and provide an iterative explanation of how the IIF developed and how it was mobilised.

\section{Why Realist Evaluation?}

Pawson and Tilley's (1997) realist evaluation offers significant potential for the SfD field. They point out that a key focus for understanding social change is to not justify 'what works' but to explain what it is about a project that makes it work or not. Pawson and Tilley emphasise the role of context in social change projects because the conditions, environments, 
socio-economic backgrounds of the participants involved in a given project will influence project outcomes (Dalkin et al. 2015). It is not possible to generalise the success of a project to another location because social projects are context dependent and they are complex. Pawson and Tilley also afford significant attention to causality, which for them is conceptualized as the 'generative' behavioural triggers and mechanisms that a project may bring about (Pawson \& Tilley 1997); placing emphasis on how reasoning, attitudes and behaviours of participants intersect with the resources of a project.

A realist approach to evidence involves understanding what works for whom, in which circumstances, and why (Pawson \& Tilley 1997). On a pragmatic level, Pawson and Tilley suggest that social projects are 'program theories' constituting assumptions and beliefs of how and why a program should work (Funnell and Rogers 2011). Thus, the first step in any evaluation is to establish a program theory for testing, made up of 'candidate' Context Mechanism - Outcome (CMO) explanations where:

$\mathrm{C}=$ What conditions are in place for a project to trigger mechanisms to produce outcome patterns?;

$\mathrm{M}=$ What is it about a project that may lead it to have a particular outcome pattern in a given context (for example, how do resources intersect with participants beliefs, reasoning, attitudes, ideas and opportunities?); and

$\mathrm{O}=$ What are the practical effects produced by causal mechanisms being triggered in a given context?

Having established a program theory, realist evaluation involves mobilising mixed methods (qualitative and quantitative) to test and establish what worked for whom, in what circumstances and why in order to lead to a refined new program theory. The scope and potential for realist evaluation's use in social change is significant, given that inputs and resources contribute to project outputs and outcomes .

The argument for realist program development and evaluation for SfD is compelling because sport may work for some people in certain circumstances in which they are often combined with other interventions and resources. Indeed, whilst sport might provide the context for positive development, understanding how and why social processes combine to explain SfD's contribution (Coalter 2013) could be a key focus.

\section{Building the IIF}


I created the IIF to build the capacity of practitioners to develop a realist program theory (Pawson \& Tilley 1997) in order to hypothesize (prior to delivery) how, why, and for whom their projects may work. Whilst delivering their projects, the IIF intended to build their capacity to evaluate and test their program theories through realist evaluation. I entirely recognised at this point that it was crucial to consider how realist evaluation could be mobilised. There were challenges around this, not to mention the limited evidence of practitioners mobilising an approach that is mainly positioned within academic discourses. I also recognised that others may not necessarily share my view that it was an appropriate methodology.

I felt that Shulha et al's (2016) CAE principles could mobilise the capacity building of realist evaluation amongst the practitioners. Shulha et al's (2016) CAE principles attempt to take context into consideration; what is needed within the evaluation; and whether the goal is practical (outcomes that foster use to inform decisions about the program under examination) or transformational (changing an individual's disposition towards learning and even evaluation practice) (Cousins, Whitmore \& Shulha, 2013). For the purposes of this application I numbered the CAE principles indicated in Box 1 (see Chapter 1 for more detail about the principles)

\section{Box 1: CAE Principles}

P1- Clarify motivation for collaboration

P2- Foster meaningful relationships

P3- Develop a shared understanding of the program

P4- Promote appropriate participatory processes

P5- Monitor and respond to resource availability

P6- Monitor evaluation progress and quality

P7- Promote evaluative thinking

P8- Follow through to realize use

Initially, I was interested in Shulha et al's appreciation of context and P1 'Clarify motivation for collaboration'. I immediately mobilised this principle within my consultation with the practitioners to establish whether realist evaluation was something they could, or would be willing to mobilise. I also consulted with academic colleagues examining each CAE principle, and their relevance to the context of the practitioners and integration in the IIF. This preliminary process uncovered no resistance from those consulted, and a case emerged for integrating the CAE principles with the application of realist evaluation. Figure 1 illustrates the IIF and its composition (Note the cross reference to CAE principles in Box 1). 


\section{Insert Figure 1 about here}

The IIF demonstrates a cyclical and fluid evaluation process of realist evaluation. The process is underpinned by five key milestones from 'Developing program theory' through to 'Communicating evaluation findings'. I supported these milestones with specific workshops that showcase certain CAE principles (e.g., P1, P2) which embodied and appreciated all of the relevant principles throughout the evaluation cycle. Naturally, some were more prominent than others at each milestone, but the time between each milestone recognised the currency of all of the CAE principles. For example, P2 'Fostering meaningful relationships' and P3 'Develop a shared understanding of the programme' clearly apply to every stage of a collaborative evaluation process.

\section{Developing program theory}

Practitioners start by researching the field through situational analysis (Funnel \& Rogers 2011) establishing needs and mapping the area in which the project will take place. The practitioners conjecture how and why their project will work for certain people in certain circumstances making use of Pawson and Tilley's CMO explanation. In order for this to be grasped by the practitioners, I mobilised a series of workshops which had the CAE principles P1, P2 and P3 firmly embedded in their delivery. Clarifying motivation for collaboration was key to understand the practitioners' circumstances and what was realistic for them to achieve, in addition to determining how transformational or useful the IIF could be for them. I intended these workshops to foster meaningful relationships (P2) where I could establish and set boundaries of our relationship. Furthermore, this milestone provided a good opportunity to begin developing a shared understanding of the program (P3) amongst myself and the practitioners and to articulate how and why their project could work.

\section{Developing realist evaluation questions}

I worked with the practitioners to focus on the key aims of their evaluation and the types of evaluation questions to develop. These aims were placed within the lens of realist evaluation which attempts to explore what it is about a project that makes it work or not (Pawson \& Tilley 1997). I argue that all of the CAE principles are considered here, however in addition to P1- P 3, P7 'Promote evaluative thinking', and P8 'Follow through to realise use', are crucial. In this workshop, the practitioners were encouraged to consider how influential they wanted the evaluation to be for their learning as well as the learning of additional stakeholders they were coming into contact with. I encouraged them to consider to 
what extent the evaluation could influence changes in practice and refinements to their project (another key pillar of realist evaluation).

\section{Agree appropriate methods to answer questions}

I worked with the practitioners within these workshops to reflect on their competency in research methodology, covering qualitative and quantitative methods (e.g., interviews, focus groups) and encouraging the practitioners to consider what methods would best suit their evaluation. P4 'Promote appropriate participatory processes' was crucial here to consider my role in guiding them to mobilise the most suitable method, but also enable them to establish control over these processes. It was also imperative to apply P5 to enable me to respond to the individual needs of the practitioners and guide them to the appropriate resources to enable the application of the methods they selected. Whilst recognising the need for shared understanding (P2) and control over the evaluation (P4), P6 'Monitor evaluation progress and quality' was also considered in order to recognise any quality assurance issues associated with the progress of the evaluation.

\section{Analysing data and findings to enhance use}

At this stage the practitioners had fully mobilised their projects in the surrounding community and conducted the agreed methods to answer the questions underpinning their realist evaluation of how and why their project had worked. Given the disparity of every project (where some mobilised different methods), each workshop was tailored to the needs of each individual practitioner making up each project. This milestone was crucial to support the practitioners in making sense of the data they collected to draw sound and credible conclusions around their findings (P5 and P6). I recognised how challenging it could be to make sense of data analysis techniques. In line with P4, I wanted to support the practitioners but balance that support so that they were in control in mobilising the methods and data analysis. P7 and 8 were illuminated again to encourage the practitioners to consider the implications of their findings for learning and influencing change.

\section{Communicating evaluation findings}

I supported the practitioners to reflect upon the findings of their realist evaluation. I asked the practitioners to consider whether the findings of their evaluation were able to make sense of how and why their project worked in a justifiable and valid way (P5, P6). By being involved throughout the collaborative process, my shared understanding of the program (P3) 
intended to help the practitioners make sense of this. I also intended to help them make decisions about how they should communicate their findings. Following through to realise use (P8) was very pertinent here, but equally, promoting evaluative thinking (P7) was of significant interest to me. It was crucial, for example, to establish whether the act of carrying out realist evaluation had enhanced any learning on levels of transformation and / or practical use (Cousins et al., 2013).

\section{Evaluating the IIF}

\section{Methodology}

My aim for evaluating the IIF was to test whether a framework that integrated CAE and realist elements could work as an appropriate approach to leverage evaluation capacity building for the practitioners involved.

The methods selected for this study were made up of Q-method (Watts \& Stenner 2012) which falls within Q methodology (Brown 1980). Q-method is a qualitative and quantitative approach that focuses on subjective viewpoints of its participants (Brown 1980; Watts \& Stenner 2012); asking them to decide what is 'meaningful' and what does (and what does not) have value and significance from their perspective (Watts \& Stenner, 2005). Qmethod involves researchers developing a set of statements representing the viewpoints of certain individuals about an issue, program or phenomena (in this case, experience with the IIF). These statements are ranked by participants relative to one another into piles of agreement (e.g., +4, +3), disagreement (e.g., -4, -3) and neutral (i.e.,. 0) by use of a 'Q grid', an example being depicted below in Figure 2 (Watts \& Stenner, 2012). The production of a Q-sort for each participant is then produced (see Figure 3 as an example of a completed sort where the numbers in each box correspond to each statement). Factor analysis is subsequently statistically implemented through the PQ method software package to identify shared correlations of viewpoints amongst the sample of individuals which creates groups of the participants with their own 'shared' overall Q-sort. The statistical data within each sub/group is qualitatively interpreted by the researcher to produce a story or holistic narrative about those shared viewpoints (Watts \& Stenner 2012).

\section{Insert Figure 2 about here}

Fifteen practitioners ranked 30 statements (that I produced to test the IIF) into the Qsort grid (see Figure 3 for an example of an individual completed sort). These 15 participants 
worked in the groups of 3 across the 5 projects ( 6 female and 9 male) to create their own individual Q-sort.

\section{Insert Figure 3 about here}

I constructed the statements in accordance with the IIF under evaluation; specifically, the capability of the IIF with its embedded CAE principles to build capacity in realist evaluation. The statements were structured across three key areas (competency and progress in evaluation), (competency in and attitudes towards realist evaluation) and (impressions about the collaborative dimension of the IIF). I wanted the statements to capture the distance travelled in evaluation capacity but also capture how the CAE principles integrated with realist evaluation. Table 1 lists the 30 statements; it is quite clear that the CAE principles are not explicitly stated as statements. I constructed the statements in this way in order to make the language easy to understand. This approach still enabled the testing of the CAE principles given their application and relation to the statements; each applicable principle is crossreferenced to each statement in Table 1 (e.g., P1, P2). You will see that more than one principle may apply to each statement which demonstrates the coverage of the principles to each statement (opposed to focusing on one pure statement in isolation, and producing an overload of statements). Before the Q-sort exercises were carried out the statements were validated in three specific ways. First, by myself through my reflexivity and engagement in mobilising the IIF to ensure that they represented what the IIF sought to achieve. Second, the statements were presented to a small sample of the practitioners to clarify that they made sense and reflected the IIF. Finally, they were cross-checked by the same academic colleagues who were consulted around the application of the CAE principles to the IIF.

\section{Insert Table 1 about here}

Once all practitioners had completed their Q-sorts, I inputted each one into a quantitative statistical program (PQ-Method), which produced a 'factor analysis' (distinct in Q-sort from commonly known and used exploratory factor analysis using, for example, Varimax rotation). The factor analysis identified 8 possible factors (groups) for qualitative interpretation across the 15 completed Q-sorts. Having examined the statistical relevance of each group, I selected four groups for full qualitative interpretation based on the criteria of more than one practitioner loading into each group and overall study variance. This was made up of 6 participants in Group 1, 4 within Group 2, 3 within Group 3, and 2 within Group 4. Those within Groups 1, 2 and 3 spanned all five CIPs, whereas those in Group 2 were representative of only a single project. I interpreted each resultant Q-sort score for each of the four groups to produce a 'holistic narrative' (Watts \& Stenner, 2012) which was informed by Watts and 
Stenner's (2012) crib sheet (Table 2). The crib sheet focused on where the statements were ranked to enhance the building of the narrative for each group; specifically focusing on how and to what extent the IIF leveraged evaluation capacity.

\section{Insert Table 2 about here}

\section{Findings}

My aim of this evaluation was to illustrate how the IIF worked to integrate realist evaluation with the CAE principles in the interest of individual evaluation capacity building. I now illustrate each holistic narrative for the four groups of practitioners that emerged from the data analysis. Again, each narrative is the result of the iterative qualitative interpretation I followed for understanding how and why the IIF worked for the practitioners making up each group. I attempt to demonstrate how the IIF worked in different ways for the practitioners across the four groups making specific reference to emerging CAE principles.

\section{Group 1 narrative: New and emerging evaluators (6 participants)}

The shared viewpoints from this group indicate that the evaluation competency of the practitioners has increased significantly from being involved in the framework which aligns with 'P7 Promote of evaluative thinking' and 'P8 Follow through to realise use'. It supports the importance of 'P4 Promoting] appropriate participatory processes' through the workshops to support the key stages of realist evaluation. The six practitioners view it as necessary, but not crucial, to have evaluation capacity building embedded in the support and development of a practitioner role which was not rated as high compared to other groups. Despite their increased competency, the practitioners are cautious about the amount of evaluation that should be embedded in their roles due to the time constraints and additional workloads. This highlights key issues around monitoring resource availability (P5) and the level at which evaluation work can be expected of practitioners in this context. However, they do feel considerably more confident about mobilising evaluation in the future which emphasises distance travelled around the promotion of evaluative thinking (P7).

Realist evaluation competency has improved significantly amongst this group which is further justified by their improved understanding of identifying key realist terms such as 'mechanisms' within their project. This is further supported with other key conceptual elements that support the realist evaluation process such as the importance of developing 
program theories of change, seeing the value in developing CMO configurations, and simply understanding more about how and why their project worked.

They ranked higher (relative to other groups) the statement associated with the framework for developing program theory which was made possible through developing a shared understanding of their project with the trainer and the others in their project. They also ranked higher than any other group the benefits of the framework for providing a clear set of steps to follow. These findings highlight the importance of developing appropriate participatory processes (P4) to provide structure and support for the contextual needs of those carrying out the evaluation.

Overall, it is quite apparent that the IFF has fostered use and increased competency of the evaluation which illustrates the significance of P8 and P4. However, whilst showing a grasp of the realist approach, they do not appear to demonstrate any critical issues or feelings about realist evaluation: this is reflected in their neutral ranking of statement 11 (appropriateness of realist evaluation), and may suggest that although heavily involved, they have relied to some extent on others to overcome issues and specific barriers imposed by the evaluation approach because they lack deeper engagement of evaluative thinking. It also stems from a motivation for collaboration that was more focused on the use of the evaluation for getting the work done. There is a lack of confidence in the findings of their evaluation having any real impact on the external landscape such as partners partly because they are not critical of that landscape. They are less likely, therefore, to follow through to realise use of the findings (P8) of their realist evaluation..

\section{Group 2 narrative: Polished problem solvers (4 participants)}

It is apparent that 'use' in evaluation is a prevailing characteristic for the four practitioners within this group. They make sense of realist evaluation in the sense of it being a suitable evaluation approach for their project. 'P8 Follow through to realise use' emerges on the basis of group members being able to understand how and why their project worked and meeting the goals of their evaluation. Actively applying the evaluation through and alongside their own project was a key factor to this which can be associated with 'P4 Promote appropriate participatory processes'. As part of understanding 'P1 Clarify motivation for collaboration', it was understood that they were more active learners; thus being able to apply realist evaluation within the context for their project was key. On a more transformational and 'P7 Promote evaluative thinking' level, these practitioners show willingness to make use of realist evaluation in the future and feel that evaluation should be part of a practitioner's 
role. The main factor in this desire relates to the clear realisation of understanding how and why their project has worked. Practitioners within this group are evaluative thinkers focused on the willingness to learn and gain more skills, which is another key motivation for collaboration. Their competency in conducting evaluation has increased (consistent with groups one and four).

In accordance with 'P1 Clarify motivation for collaboration' and 'P4 Promote evaluative thinking', the practitioners are more conceptually engaged in SfD and realist evaluation which may explain their desire to continue mobilising it in the future. This engagement is supported through the way they make use of key resources to support their learning within the framework and enables them to solve problems, which is confirmed given their increased reflexivity and recognition of the limitations imposed by the realist evaluation. In particular, they do not place significant approval towards the CMO configuration, yet they make attempts to address these issues through improvisation and 'P3 Develop[ing] shared understanding of the programme' with those with whom they are working. 'P4 Promot[ing] meaningful relationships' with those around them (including a positive relationship with the trainer) enabled this problem solving and increased confidence. Thus ' $\mathrm{P} 7$ Promote evaluative thinking' is slightly quashed given their limited confidence as to where their findings will go. Despite the new fostering of evaluative thinking for mobilising realist evaluation, they are still apprehensive about carrying out evaluation in the future mainly due to the limited perception of how well regarded their findings will be. This is also supported by the concerns they have over time to imbed such work which raises implications for 'P5 Monitor and respond to resource availability'.

Overall, the practitioners are keen for guidance and support and use that 'P3 Develop[ing] shared understanding of the program' to enable effective evaluation mobilisation and competency. Structure and guidance is needed and relied upon because of their limited experience in carrying out evaluation. However, what distinguishes this group compared to others is the deeper reflexivity and engagement towards realist evaluation, coupled with their ability to solve problems. This has illustrated 'P7 Promote evaluative thinking', and 'P8 Follow through to realising use'. Having a pre - defined framework (IIF) has facilitated an 'P4 Promote appropriate participatory process', enabling the practitioners to mobilise their evaluation from the outset (based on their 'P1 Clarif[ication of] motivation for collaboration') as opposed to the view of building it entirely themselves and choosing from the multitude of other approaches. 


\section{Group 3 narrative: Passive Passengers (3 practitioners)}

The practitioners within this group share viewpoints that evaluation should be a key part of a practitioner's role and continual professional development, which suggests transformational characteristics in that evaluation work should be centred within a practitioner's role. Such was more prominent in this grouping than any other.

However, despite this evidence of 'P7 Promote of evaluative thinking', deeper interpretation of this group indicates that the practitioners are less critical of the evaluation context for SfD landscape and perhaps do not appreciate the extent of the challenges for practitioners taking this approach. For example, they are less reflexive than those in Groups 1 and 4 about their approach to evaluation and appear less engaged. This is further supported by their ranking of other statements suggesting that they may not be as inclined to carry out evaluation in the future, and specifically realist approaches. On that basis, 'P8 Follow through to realise use' and the 'P7 Promote of evaluative thinking' is not as pertinent compared to other groups. In similar respects to Groups 2 and 1, there is little confidence of their evaluation findings having any impact. However, in this case, this is predominantly due to their weaker reflexivity towards evaluation and being aware of the wider outlets for such dissemination.

These practitioners have perhaps taken more of a surface level approach to evaluation where the process has partially 'P8 Follow through to realise use' but that use has been more reflected in the reliance and encouragement from other practitioners in their project. This highlights a 'P1 Clarify motivation for collaboration' situated more within the surface approach to engaging in the IIF opposed to anything transformational. Practitioners within this group find it challenging to see the connection as to how the learning and deeper understanding of their project can be synthesised with evaluation findings. As such, potential issues arise around 'P4 Promote appropriate participatory processes' because a key focus of the IIF was to provide a real time opportunity to contextualise the evaluation alongside their CIPs as practitioners.

Realist evaluation competency is not significant to this group nor is there any specific intention to carry it out in the future because of issues with grasping its terminology and jargon. This is further supported by the disengagement with the resources provided and stages to follow in the IIF in the evaluation cycle.

Overall, whilst these practitioners feel that they have mobilised the evaluation, their engagement is much less evident as compared to other groups which does not highlight any 
significant currency in 'P7 Promote evaluative thinking' or 'P8 Follow through to realise use'. These practitioners appear more 'laid back' about the IIF as opposed to other groups and seem content to be guided by others, which illustrates the need for deeper consideration of 'P5 Monitor e and respond to resource availability' alongside 'P6 Monitor evaluation progress and quality'.

\section{Group 4 narrative: Proficient yet sceptical (2 practitioners, each in the same project)}

The shared viewpoints of those within this group suggest that the IIF has facilitated the mobilising of their evaluation. Without the structure of the framework and opportunity to apply it in practice they would have found the process of evaluating their project challenging. This illustrates the IIF's approach for 'P4 Promote appropriate participatory processes'. These practitioners share the views that the relationship with the trainer enabled deeper understanding of the evaluation process within a strong context of ' $\mathrm{P} 4$ Foster meaningful relationships'. There is a mutual sense of 'P3 Develop a shared understanding of the program' which enables this grasping of evaluation. Trust is maintained whereby these group members feel they are able to shape their evaluation design within through 'P4 Promote appropriate participatory processes' of the IIF.

These practitioners assert that they do not see themselves in significant control of the evaluation which puts limits on 'P4 Foster appropriate relationships' or 'P3 Develop a shared understanding of the program' CAE principles underpinning the framework. For them, the context of their project, which was often subject to change, and working with very young people made it hard for them to mobilise the evaluation as they initially intended.

Those in this group express viewpoints that do not directly favour realist evaluation as an evaluation approach. They are less likely than any other group to carry out realist evaluation in the future mainly because of their apprehension towards developing and testing program theory within the context of their project. Although their grasp of 'P1 Clarify motivation for collaboration' was positive from the outset and throughout, the context of their project and sample group (which were young children aged 5-7) made it very challenging to mobilise realist evaluation. Although, through their very recognition of these limitations of realist evaluation, these practitioners show signs of 'P7 Promote evaluative thinking' and awareness of 'P8 Follow through to realize use', be it positive or negative.

Furthermore, on a transformational level, they more than any other group feel that their findings will be valued by others highlighting how the IIF has associated with 'P8 
Follow through to realis use'. Group members felt that their findings are likely to have an impact beyond the micro level of their project itself because the approach taken has uncovered new knowledge, which suggests that realist evaluation has been able to evoke findings that will be recognised externally and influence future practice within the workings of partnering stakeholders.

Therefore, in conclusion, this group is interesting because members have seen the mobilising of realist evaluation as a challenge, yet have been able to see the potential benefit it brings for informing learning and future practice. There are strong signs of 'P7 Promote evaluative thinking' and 'P8 Follow through to realise use' in this group. Despite their apprehension to mobilise realist approaches in the future, it is apparent that the IIF and its stages of delivery, coupled with the support of the trainer, adequately touched on 'P4 Promote appropriate participatory process'.

\section{Discussion and Conclusions}

\section{Summary of findings}

In this chapter I have drawn upon the key findings that emerged from my application of the IIF that attempted to integrate elements of both Shulha et al's. (2016) CAE principles and realist evaluation (Pawson \& Tilley, 1997). To reiterate, I developed the IIF to support 15 practitioners to use realist evaluation to evaluate their own SfD projects. The key aim for the IIF was to leverage evaluation capacity building among the practitioners involved in the framework. The narratives have illustrated how the IIF has worked in different ways for the practitioners working across the five projects illuminating the relevance of specific CAE principles for leveraging of evaluation capacity.

Those within Group 1 travelled the furthest in their evaluation competency; made possible through the establishing of 'P4 Promote appropriate participatory processes'. However, they required strong support and guidance through 'P2 Fostering meaningful relationships' to build capacity. 'P1 Clarify motivation for collaboration' for those in this group was much more focused around 'P8 Follow through to realise use'. Those within Group 2 required support and guidance like those preceding them, which was made possible through the similar CAE principles around relationships and appropriate processes. However, they were more capable in dealing with specific issues which linked very strongly to their intention to 'Follow through to realise use' for the future and the 'P7 Promote evaluative thinking'. The same can also be said for Group 4, yet with more apprehension around the 
application of realist evaluation on a transformational level. Despite this apprehension, they were still able to see the influence of realist evaluation for their project illustrating evaluative thinking. Group 3's engagement within the IIF was far more surface level when compared to the other groups as their motivation for collaboration was uniquely different to other groups. Group 3 were far more reliant on others to fulfil the evaluation as they were working in their projects across the other groups. This raises particular issues around the different levels of motivation amongst practitioners working in teams within projects. For example, as this evaluation has shown, different practitioners may embody different levels of engagement with evaluation practice.

\section{To what extent do CAE principles leverage capacity?}

Applying the CAE principles to this process has been a valuable asset to see how there are different applications of the principles within each subgroup. Apart from Group 3, it is quite clear that the IIF has leveraged capacity building in realist evaluation. However, as promoted in 'P1 Clarify motivation for collaboration', context is crucial for understanding how the CAE principles will unravel in different ways. For example, P1 applied to different groups in different ways: in Group 1 motivation for collaboration was primarily focused on use, and in Group 2 there was more of a transformational focus. This highlights the significance of P1 regarding the importance to establish motivation for collaboration which emphasises the importance of grasping key contexts around practitioner motivation. Across the groups, there were similarities across the principles: namely, P7 where the framework was able to promote evaluative thinking. For some groups (particularly Group 2), P8 on following through to realise use was pertinent as there was specific intention to continue with a similar application of the IIF in the future. This demonstrates significant transformational characteristics and depth in evaluative thinking. Despite apparent nuances in each group, there was clear synergy across P2 which focused on the importance of a meaningful relationships that provided support and structure whilst respecting the independent role of the practitioners.

The positive integration of realist evaluation with CAE principles show potential for developing and fostering realist methodologies for evaluation with practitioners on the ground. It is nevertheless important to recognise that the framework should be mobilised in accordance with the contextual needs of those who may be using it. 
The integration of the CAE principles with realist evaluation within the IIF has illuminated where they emerge throughout the collaborative evaluation process. Whilst some have emerged more than others within the narratives, all are crucially embedded within the IIF. They have been key guiding principles for the mobilising of realist evaluation. A key strength of the CAE principles is the way they are able to capture all of the dynamics of the collaborative evaluation process.

\section{Limitations}

It is important to acknowledge the potential limitations connected with this evaluation. The first limitation concerns my involvement within the context of the IIF. For example, it is important to recognise my position as 'Course Leader' within the practitioners' curriculum, and my role as the developer and trainer within the IIF, as well as the evaluator of the IIF itself. This position could be open to challenge around the areas of 'bias', particularly given my close relationship with the students and, perhaps, their inclination to tell me what I wanted to hear. However, Q-method was able to mitigate this on a number of levels. First, the Q-sort activity did not involve me interviewing or questioning the practitioners in any overly influential way. The development and validation of the statements simply involved me presenting the statements to the practitioners which I allowed them to sort in their own space and privacy, and thus to feel more open and free as to how they might respond. The software package of PQ method then quantitatively ran the factor analysis for shared viewpoints to create the groups.

As 'trainer' in the IIF, I contend that such a close engagement enabled me to see an even clearer integration of the principles with realist evaluation and support 'P2 Foster meaningful relationships'. Each practitioner understood they could opt in or out of the IIF at any time and thus potential issues around trainer-practitioner relationships were evaded, and motivation for collaboration was open and transparent. Overall, it would go against the very philosophy of practitioners mobilising evaluation independently if I were to stress the importance of externalising the evaluation of the IIF to someone else (Adams \& Harris, 2014).

\section{Implications for CAE and ongoing use of IIF for evaluation capacity building in SfD}


A question residing over work of this nature concerns the extent to which practitioners have the capacity to conduct evaluation. Given the context of the lack of evidence, I have asserted a rationale for involving them more closely within a collaborative evaluation process, underpinned by the IIF. The findings informing this evaluation demonstrate that the IIF has capacity for enabling practitioners to collect and analyse their own data and produce findings and it should be tested further in other contexts. This is important for SfD, given the context of an evidence base for sport which is currently under developed (Edwards 2015). This demonstrates the role that practitioners may be able to have in the process, and advocates the potential of realist approaches for understanding how and why programs work.

However, my findings have demonstrated how important it is to consider how much work we should give practitioners to do and how realistic it may be to mobilise evaluation on a practitioner level within a recognition of resource and capacity constraints. I propose that the next appropriate steps for mobilising the IIF in SfD would be to work with different and more experienced practitioners in order to start developing frameworks from the outset in collaboration with the practitioners. Practitioners could be involved in shaping the approach and evaluation methodology, and could rate and shape the relevance of each CAE principle to the collaboration and where each one should apply. However, this would be somewhat dependent on motivation for collaboration and context.

Moreover, as the results identify, the role of the CAE principles are essential for building such capacity and have offered substantial insight in understanding how and why the framework worked for different practitioners. The CAE principles offer strength across so many levels (as can be seen in their application in the IIF) and offer considerable value around 'P1 Clarify motivation for collaboration' in determining why and on what grounds practitioners engage in evaluation practice. Additionally, they provide focus around the design of the participatory process taking into consideration resource needs; they consider the importance of developing meaningful relationships; and they consider the design of the evaluation whilst acknowledging that any process may or may not foster transformational learning alongside practical use. Evaluators embracing the CAE principles may be able to open up and establish key parameters around 'use' and 'transformational' aspects of how given CAE should unfold. 


\section{References}

Abhyanker, P, Cheyne, H, Maxwell, M, Harris, F \& Mcourt, C. (2013). A realist evaluation of a normal birth programme. Evidence based midwifery, 11 (4), 112-119

Adams, A. (2008). 'Building Organisational and Management Capacity for the Delivery of Sports Development', in: V. Girginov, (ed.), Management of Sports Development. Oxford: Butterworth-Heinemann, pp.203-225

Adams, A., \& Harris, K. (2014). Making sense of the lack of evidence discourse, power and knowledge in the field of sport for development. International Journal of Public Sector Management, 27 (2), 140-151

Brown, S.R. (1980). Political Subjectivity: Applications of Q Methodology in Political Science. New Haven, CT: Yale University Press

Coalter, F. (2007). A wider social role for sport. London: Routledge

Coalter, F. (2010). The politics of sport-for-development: Limited focus programmes and broad gauge problems? International Review for the Sociology of Sport, 45 (3), 295-320

Coalter, F. (2013). Sport for Development what game are we playing? London: Routledge

Cousins, J. B., Whitmore, E., \& Shulha, L. (2013). Arguments for a common set of principles for collaborative inquiry in evaluation. American Journal of Evaluation, 34, 7-22

Crabbe, T. (2007). Positive Futures: Putting the Pieces Together? - The 2007 Annual Positive Futures Monitoring and Evaluation Report

Dalkin, S; Greenhalgh, J; Jones, D; Cunningham, B; \& L hussier, M. (2015). What's in a mechanism? Development of a key concept in realist evaluation. Implementation Science, 10 (49), 1-7

Duffy, P., North, J., \& Muir, B. (2013). Understanding the impact of sport coaching on legacy. International Journal of Sport Policy and Politics, 5(2), 165-182. $10.1080 / 19406940.2012 .665380$

Edwards, M. (2015). The role of sport in community capacity building: An examination of sport for development research and practice. Sport Management Review, 18(1), 6. https://search.proquest.com/docview/1655112292

Fetterman, D. M. (2001). Foundations of empowerment evaluation. Thousand Oaks, Calif. [u.a.]: Sage. 
Funnell, S. \& Rogers, P (2011). Purposeful program theory. Affective use of theory of change and logic models. Jossey Bass.

Harris, K. (2018). Building sport for development practitioners' capacity for undertaking monitoring and evaluation - reflections on a training program building capacity in realist evaluation. International Journal of Sport Policy and Politics. https://doi.org/10.1080/19406940.2018.1442870

Harris, K. \& Adams, A., (2016). Power and discourse in the politics of evidence in sport for development. Sport Management Review, 19 (2), 97-106

Hylton, K and Hartley, H.J. (2011). 'Sports development... a profession in waiting?'. In S. Harris, B. Bell, A. Adams and C. Mackintosh (Ed.), Sport for sport? practical and theoretical insights in sports development. Brighton: LSA Publication 117.

Jagosh, J., Macaulay, A., Pluye, P., Salsberg, J., Bush, P., Henderson, J.G,T.

(2012). Uncovering the benefits of participatory research: Implications of a realist review for health research and practice. The Milbank Quarterly, 90(2), 311-346. 10.1111/j.1468-0009.2012.00665.

Kay, T. (2009). Developing through sport: Evidencing sport impacts on young people. Sport in Society, 12 (9), 1177-1191

Kay, T. (2012). Accounting for legacy: Monitoring and evaluation in sport in development relationships. Sport in Society, 15(6), 888-904. 10.1080/17430437.2012.70828

Levermore (2011). Evaluating sport-for-development: approaches and critical issues. Progress in Development Studies, 11 (4), 339-53

Mwaanga, O. (2011). Understanding and Improving Sport Empowerment for People Living with HIV/AIDS in Zambia. Unpublished PhD Thesis

Nicholls, G. (2007). 'Reflections on researching the ability of sports interventions to reduce youth crime - the hope of scientific realism', in: K. Hylton, J. Long and A. Flintoff (ed.), Evaluating Sport and Active Leisure for Young People LSA Publication No. 88 ISBN: 978-0-906337-99-8

Nicholls, S, Giles, A \& Sethna, C. (2010). Perpetuating the 'lack of evidence' discourse in sport for development: Privileged voices, unheard stories and subjugated knowledge. International Review for the Sociology of Sport. 46 (3), 249-264

Pawson, R \& Tilley, N. (1997). Realistic Evaluation. Sage 
Shulha, L, Whitmore, E, Cousins, B, Gilbert, N \& Hudib, H (2016). Introducing EvidenceBased principles to guide collaborative approaches to evaluation: Results of an Empirical process. American Journal of Evaluation, 37 (2), 193-215

Smith, A., \& Leach, R (2010). 'Evidence. What evidence?': evidence-based policy making and School Sport Partnerships in North West England. International Journal of Sport Policy and Politics, 2 (3), 327-345

Watts, S. \& Stenner, P. (2005). Doing Q methodology: theory, method and interpretation. Qualitative Research in Psychology, 2 (1), 67-91

Watts, S. \& Stenner, P. (2012). Doing Q methodological research: Theory, method \& interpretation. Sage

Weed, M. (2016). Should we privilege sport for health? The comparative effectiveness of UK Government investment in sport as a public health intervention, International Journal of Sport Policy and Politics, 8, (4), 559-576 
Figure 1: IIF Framework (P1 through P8 represent specific CAE principles - see Box 1)

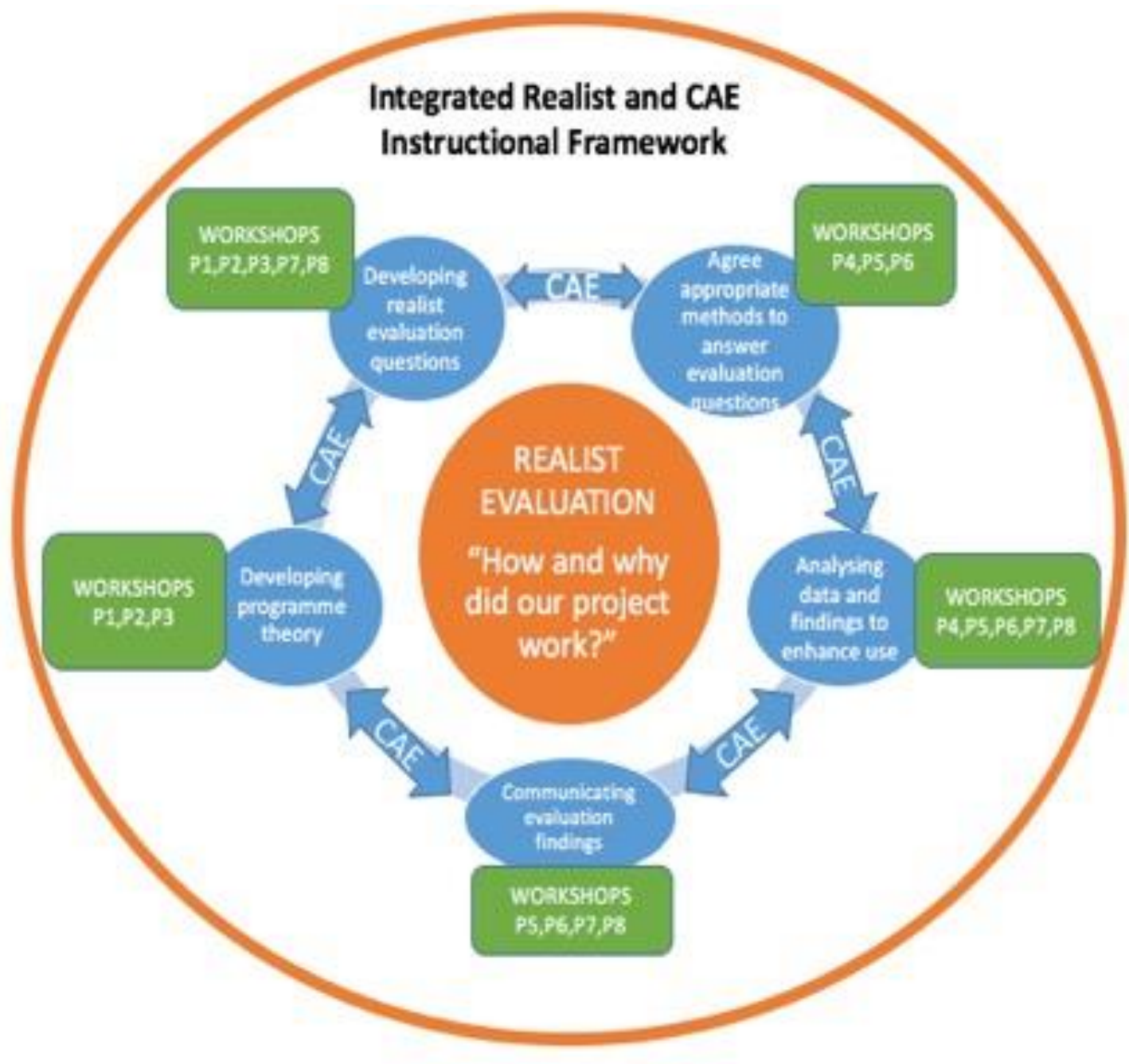


Figure 2: Example Q Grid used in testing

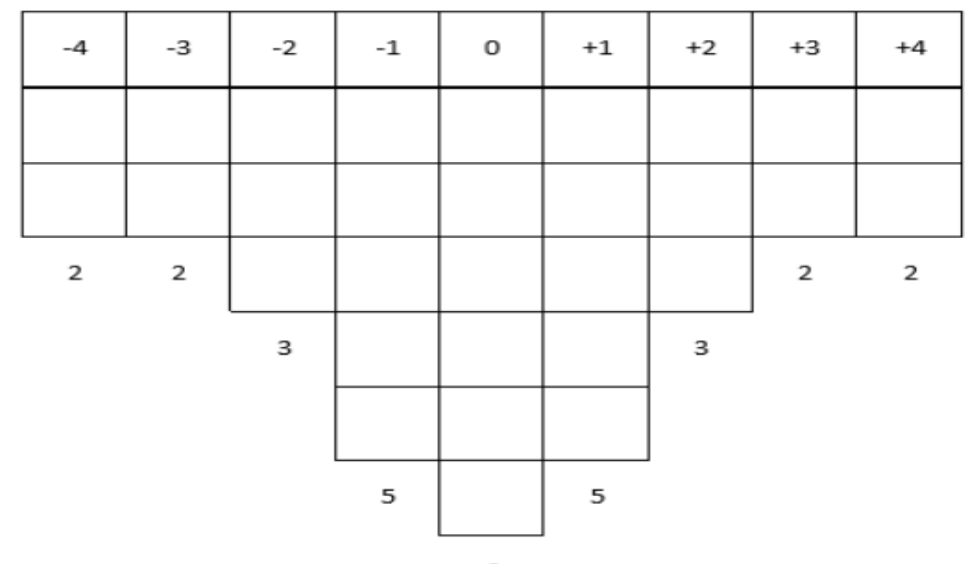


Figure 3: Example 'completed' Q Grid post sort

\begin{tabular}{|c|c|c|c|c|c|c|c|c|}
\hline-4 & -3 & -2 & -1 & 0 & +1 & +2 & +3 & +4 \\
\hline 1 & 24 & 18 & 6 & 25 & 15 & 26 & 16 & 11 \\
\hline 12 & 19 & 5 & 23 & 27 & 7 & 20 & 10 & 2 \\
\hline \multirow[t]{4}{*}{2} & 2 & 29 & 30 & 8 & 14 & 17 & 2 & 2 \\
\hline & & 3 & 28 & 22 & 3 & 3 & & \\
\hline & & & 13 & 4 & 21 & & & \\
\hline & & & 5 & 9 & 5 & & & \\
\hline
\end{tabular}


Table 1: Q-set Statements and Alignment to each CAE Principle (e.g., P6)

\begin{tabular}{|c|c|}
\hline 1. My competency for carrying out evaluation has improved (P7, P8) & $\begin{array}{l}\text { 16. The resources (eg literature) surrounding realist evaluation enabled my understanding of the } \\
\text { evaluation methodology (P4, P5) }\end{array}$ \\
\hline $\begin{array}{l}\text { 2. Since the framework I feel more competent about undertaking evaluation in the future (P4, } \\
\text { P5, P7, P8) }\end{array}$ & $\begin{array}{l}\text { 17. I felt that my relationship with the trainer enabled me to progress to understand how to } \\
\text { evaluate my project (P1, P2, P4, P7) }\end{array}$ \\
\hline 3. Since the framework I feel more competent in carrying out Realistic Evaluation (P7, P8) & 18. I feel that the structure to the framework enabled me to carry out my evaluation (P4, P5, P6) \\
\hline $\begin{array}{l}\text { 4. I can see the value in developing context - mechanism - outcome configurations to aid my } \\
\text { evaluation }(\mathrm{P} 3, \mathrm{P} 4, \mathrm{P} 7)\end{array}$ & 19. I felt engaged in the evaluation (P1, P2, P5) \\
\hline $\begin{array}{l}\text { 5. I feel that the framework has made it possible for me to identify mechanisms of change in } \\
\text { my project }(\mathrm{P} 3, \mathrm{P} 7, \mathrm{P} 8)\end{array}$ & 20. I felt that my views within the collaborative process were respected (P1, P2, P3, P4) \\
\hline $\begin{array}{l}\text { 6. I think that it is important to develop a programme theory before an evaluation is carried out } \\
(\mathrm{P} 3, \mathrm{P} 4)\end{array}$ & 21. I felt that throughout the framework I was able to highlight any issues or tensions (P2, P3, P6) \\
\hline $\begin{array}{l}\text { 7. Developing a programme theory stage enabled me to explain my programme theory } \\
\text { effectively }(\mathrm{P} 3, \mathrm{P} 4, \mathrm{P} 7)\end{array}$ & 22. My role within the evaluation was clear $(\mathrm{P} 3, \mathrm{P} 6)$ \\
\hline $\begin{array}{l}\text { 8. The framework provided me with a clear set of steps to follow for conducting a realistic } \\
\text { evaluation }(\mathrm{P} 4, \mathrm{P} 7)\end{array}$ & 23.The trainer and I shared the understanding of the evaluation goals we had (P1, P2, P3, P7) \\
\hline $\begin{array}{l}\text { 9. I am more reflexive now about the processes I take to evaluate programmes / projects (P4, } \\
\text { P7, P8) }\end{array}$ & 24. I feel that the findings from our evaluation will be valued by our partners (P3, P5, P6, P7, P8) \\
\hline $\begin{array}{l}\text { 10. Working with my fellow students in my project enabled me to understand realist } \\
\text { evaluation (P2, P3, P5) }\end{array}$ & 25. The framework was suitable to meet the needs of my project (P1, P2, P4) \\
\hline $\begin{array}{l}\text { 11. I feel that realist evaluation is an appropriate evaluation methodology for my project (P3, } \\
\text { P4, P7, P8) }\end{array}$ & 26. I was able to be embed the knowledge of my project within the framework (P2, P3, P4, P5) \\
\hline 12. I now understand more about how and why my project worked (P3, P4 P7, P8) & 27. Throughout the framework I felt in control of the evaluation (P2, P4, P5) \\
\hline $\begin{array}{l}\text { 13. Since the framework I can see the need to understand how and why sport for development } \\
\text { programmes work (P3, P7, P8) }\end{array}$ & $\begin{array}{l}\text { 28. Without the structure of the framework I would not have been able to mobilise realistic } \\
\text { evaluation (P4) }\end{array}$ \\
\hline 14. I would implement realist evaluation in future roles (P5, P7, P8) & 29. The framework has been possible to complete given my additional workload (P1, P2, P4) \\
\hline $\begin{array}{l}\text { 15. I feel that evaluation training should be a key part of a practitioners professional } \\
\text { development (P4, P7, P8) }\end{array}$ & 30. Evaluation should be embedded within a practitioner's role (P8) \\
\hline
\end{tabular}


Table 2 Heuristics used to interpret data (Watts and Stenner 2012)

\begin{tabular}{|l}
\hline Qualitative interpretation for each group \\
$\square \quad$ Statements ranked at $+4 /+3$ \\
$\square \quad$ Statements ranked higher in the factor group than any other group \\
$\square \quad$ Statements ranked at -4 / -3 \\
$\square \quad$ Distinguishing statements \\
$\square \quad$ Using demographical information about participants and micro or macro circumstances \\
$\square \quad$ First take - (building a provisional story or theory) \\
$\square \quad$ Any other additional information from statement rankings not yet covered \\
$\square \quad$ Final take - (presenting a holistic narrative about the group) \\
\end{tabular}

